\title{
Promoção da saúde para pessoas no regime semiaberto do sistema penitenciário: relato de experiência
}

\author{
Health promotion for people in the semi-open prison system: a case \\ study
}

Anne Evelyn Gomes Serra', Reângela Cintia Rodrigues de Oliveira Lima²

DOI: 10.1590/0103-1104201912322

RESUMO Relatou-se a experiência da profissional de enfermagem na execução de ações de promoção da saúde, por meio da atuação de uma equipe multiprofissional, para pessoas que cumprem o regime semiaberto em município do interior do Ceará. Estudo descritivo, do tipo relato de experiência, realizado a partir de relatórios mensais e com auxílio de um diário de campo. Acredita-se que, apesar da implantação de políticas públicas voltadas para a população privada de liberdade, ainda existe muita dificuldade em sua implementação. Observa-se um contexto ainda muito marcado por uma assistência em saúde precária e, em alguns casos, inexistente, além de estigma e preconceito com essa população. É importante instigar discussões sobre saúde no sistema penitenciário nos centros educacionais formadores de recursos humanos para o Sistema Único de Saúde, tendo em vista a apreensão de conhecimentos e o desenvolvimento de habilidades e competências, a fim de contribuir para a adequada atuação dos profissionais de enfermagem e demais trabalhadores da saúde nesse cenário.

PALAVRAS-CHAVE Saúde do homem. Promoção da saúde. Educação em saúde. População institucionalizada.

\begin{abstract}
The Nursing professional experience in the implementation of health promotion actions, through the performance of a multidisciplinary team, for people who meet the semi-open regime in city in the interior of Ceará was reported. Descriptive study, of case studies type, carried out from monthly reports and with the help of a field diary. It is believed that, despite the implementation of public policies aimed at the population deprived of freedom, there is still a lot of difficulty in their implementation. A context that is still very marked by precarious health care is observed and, in some cases, non-existent, in addition to stigma and prejudice against this population. It is important to instigate discussions on health care in the prison system in educational centers trainers of human resources for the Unified Health System, with a view to apprehending knowledge and the development of skills and competencies, in order to contribute to the proper performance of nursing professionals and other health workers in this scenario.
\end{abstract}

KEYWORDS Men's health. Health promotion. Health education. Institutionalized population. 


\section{Introdução}

Os direitos sociais, previstos na Constituição Federal (CF), não foram implementados de forma simultânea à população brasileira ${ }^{1}$. A exemplo, tem-se o direito à saúde assegurado pelo artigo 196 da CF como um direito de todos e dever do Estado, e pelas Leis n ${ }^{\circ} 8.080 / 90$ e n ${ }^{\circ}$ 8.142/90, que regulamentam o Sistema Único de Saúde (SUS), deixando lacunas na garantia de ações efetivas de promoção, prevenção e reabilitação da saúde às Pessoas Privadas de Liberdade (PPL) ou que estejam em regime semiaberto das penitenciárias ${ }^{2}$. Assim, com o objetivo de orientar a reintegração social do prisioneiro, somente nos últimos anos, foram adotadas medidas governamentais para cumprir o dever do Estado na assistência à saúde, conforme disposto na Lei de Execução Penal (LEP) no 7.210, de 1984.

A LEP traz no capítulo II, seção III, o direito do preso e do internado de caráter preventivo e curativo à assistência à saúde, compreendendo atendimento médico, farmacêutico e odontológico ${ }^{3}$. Em 1995, a Organização Mundial da Saúde (OMS) iniciou o projeto de saúde no sistema prisional com o objetivo de promover a saúde pública e os cuidados nas prisões, bem como de facilitar a interlocução entre o sistema prisional e os sistemas de saúde. As ações do projeto envolvem o controle às doenças transmissíveis, sobretudo a tuberculose, o Vírus da Imunodeficiência Humana (HIV), a hepatite, o uso de drogas ilícitas e a redução de danos, e a saúde mental ${ }^{4}$.

Foi estabelecido pela Portaria Interministerial $n^{0} 1.777 / 2003$, dos Ministérios da Justiça e da Saúde, o Plano Nacional de Saúde no Sistema Penitenciário (PNSSP), implementado numa lógica de atenção à saúde baseada nos princípios do SUS, objetivando promover atenção integral à população privada de liberdade ${ }^{5}$. Em 2014, foi criada a Política Nacional de Atenção Integral à Saúde das Pessoas Privadas de Liberdade no Sistema Prisional (PNAISP), objetivando o direito à saúde e a garantia do acesso desse público ao SUS, respeitando os preceitos dos direitos humanos e de cidadania ${ }^{6}$. Porém, mesmo existindo um arcabouço jurídico para legitimar os diretos à população encarcerada, a implementação da atenção à saúde na prática do sistema e das instituições prisionais ainda é um desafio.

No cenário do encarceramento, as elevadas taxas de doenças infecciosas, como: HIV, sífilis, hepatite $\mathrm{B}$, tuberculose, pneumonia, entre outras, conferem ao sistema prisional o status de problema de saúde pública em potencial $^{7}$. As condições insalubres nos ambientes prisionais, como alimentação de má qualidade, estrutura física inadequada, com ventilação e iluminação precárias, celas lotadas, ociosidade, violência e dificuldade de acesso aos serviços de saúde, contribuem para situações de vulnerabilidade com relação à aquisição de agravos à saúde?

Em uma pesquisa acerca das condições de vida e saúde dos presos, foi revelado que parte deles já chega na prisão com problemas de saúde, entretanto, a queixa é de que, no cárcere, eles não são cuidados, têm poucas oportunidades de se prevenir e não há uma preocupação com a sua situação. Como resultados, revelou-se que a superlotação, o ócio, escassez de perspectivas, maus tratos e relacionamentos conflituosos afetam indiretamente a saúde ${ }^{8}$.

A atenção básica caracteriza-se por ações de saúde, desde a promoção e a proteção da saúde, a prevenção de agravos, o diagnóstico, o tratamento, a reabilitação, a redução de danos até a manutenção da saúde, permitindo uma atenção integral que impacte a situação de saúde e a autonomia das pessoas e os determinantes e condicionantes de saúde das coletividades ${ }^{9}$. É desenvolvida através de equipes multiprofissionais, em territórios definidos e perto da vida das pessoas ${ }^{\mathbf{1 0}}$. Assim, segundo a PNAISP, cada unidade prisional deverá possuir uma unidade básica de saúde prisional, e, caso inexista ambiência, a unidade do território poderá se responsabilizar por essas ações ${ }^{6}$.

Acredita-se que as PPL têm acesso restrito a bens e serviços, poucas oportunidades e maior vulnerabilidade social, devido à posição que 
ocupam na sociedade ${ }^{\mathbf{8}}$. Dentro do presídio, a equipe da unidade básica do município do estudo deve realizar campanhas anuais de vacinação, atendimento médico, odontológico, de enfermagem, entre outros, acompanhamento de doenças como tuberculose, hanseníase, diabetes e hipertensão, como também realização de exames, verificação de sinais vitais e administração de medicamentos. As ações realizadas são registradas em ata e encaminhadas mensalmente para apreciação judicial.

Destarte, o cuidado em saúde desenvolvido para a PPL, além de não dar conta de suas reais necessidades, quando realizadas, há uma predominância do modelo biomédico curativista" ${ }^{11}$. Portanto, realizar ações que deem conta de abordar a promoção da saúde e a prevenção de doenças das pessoas em regime semiaberto se torna um imperativo ético e um desafio, no sentido de enfocar a prevenção primária, agindo antes da ocorrência de situações e de agravos desfavoráveis e com a intenção de manter o respeito e o cuidado dedicados a essas pessoas. Assim, o presente artigo tem o objetivo de relatar a experiência de uma profissional de enfermagem na execução de ações para a promoção da saúde, por meio da atuação com a equipe multiprofissional, às pessoas que cumprem o regime semiaberto em um munícipio do interior do Ceará.

\section{Métodos}

Trata-se de um estudo descritivo, do tipo relato de experiência, a partir da vivência de uma equipe de residentes da Escola de Saúde Pública do Ceará, com ênfase em saúde da família e comunidade, no qual foram utilizados os relatórios mensais e diários de campo padronizados para o registro das atividades realizadas na residência.

O relato de experiência é um tipo de fonte de informação dedicada à coleta de depoimentos e registro de situações e casos relevantes que ocorreram durante a implementação de um programa, projeto ou em uma dada situação-problema ${ }^{13}$. A pesquisa qualitativa faz a análise das expressões humanas presentes nas relações, nos sujeitos e nas representações ${ }^{\mathbf{1 2}}$.

O diário de campo é muito utilizado nas pesquisas em saúde como caderno de notas no qual o pesquisador registra as conversas informais, observações do comportamento durante as falas, reações dos participantes diante dos quesitos investigados e impressões pessoais que podem ser alteradas com o passar do tempo ${ }^{\mathbf{1 2}}$.

Este relato usou alguns itens como guia de orientação dos Critérios Consolidados para Relato de Pesquisa Qualitativa (Coreq). Contém 32 itens que foram elaborados para auxiliar os pesquisadores a relatar aspectos importantes da equipe de pesquisa, métodos de estudo, contexto do estudo, descobertas, análises e interpretações ${ }^{\mathbf{1 4}}$.

O estudo também foi realizado a partir de uma perspectiva construtivista, valorizando o conhecimento prévio dos participantes. Na abordagem vygotskyana, o homem é considerado como alguém que transforma e é transformado nas relações que são estabelecidas na sociedade, onde acontece uma interação entre o ser humano, o meio cultural e social onde este se insere ${ }^{15}$. $O$ ser humano está em constante aprendizado e transformação, fruto de suas interações sociais. Seguindo essa mesma lógica, o pensamento construtivista defende a ideia de que o conhecimento é constituído pela interação do indivíduo com o meio, com o mundo das relações sociais e pela força de sua ação ${ }^{15}$.

O estudo foi realizado em uma escola municipal localizada na zona urbana do município de Tauá, Ceará. Em virtude de a escola ter uma localização mais próxima ao presídio, ela foi selecionada para o desenvolvimento das atividades de ensino na modalidade Educação de Jovens e Adultos (EJA) para a população privada de liberdade.

O município de Tauá está localizado no sertão dos Inhamuns. É sede da $14^{a}$ Região de Saúde. Tem sua rede de serviços composta por: 19 Unidades Básicas de Saúde (UBS), com 25 Estratégias Saúde da Família (ESF), um 
hospital e maternidade, uma Unidade de Pronto Atendimento (UPA), um Centro de Atenção Psicossocial (Caps) tipo II, um Centro de Atenção Psicossocial Álcool e Drogas (Caps AD), uma policlínica, um Centro de Especialidades Odontológicas (CEO), um Laboratório Central de Saúde Pública (Lacen) e um Serviço de Atenção Móvel de Urgência (Samu).

Entre as ações que os residentes em saúde da família e comunidade realizaram para a promoção e a educação em saúde está o projeto com pessoas do regime semiaberto, idealizado pela $1^{\mathrm{a}}$ vara da justiça do município, que contou com a parceria dos profissionais residentes com o objetivo de, entre outras coisas, alcançar melhor êxito na ressocialização daqueles que cumprem pena, através da educação. Assim, foi realizada uma reunião entre os residentes e a professora responsável pelo ensino dos apenados, que explicou o planejamento das atividades e orientou sobre a necessidade da construção de um projeto explicando as ações que seriam desenvolvidas com os apenados, datas e período das atividades propostas, para apreciação da juíza municipal.

As pessoas em regime semiaberto que realizam a educação básica têm faixa etária variável a partir de 18 anos, devendo cumprir uma carga horária semanal de 12 horas, distribuídas de segunda a quinta-feira no turno noturno. A turma é constituída apenas por integrantes do sexo masculino, num total de 12 pessoas. As mulheres não participaram desse projeto, pois são de responsabilidade de outra vara da justiça.

Após parecer judicial favorável, foi iniciado o grupo com os apenados. As ações educativas foram realizadas no período de outubro a dezembro de 2017, com uma duração de 90 minutos, às terças-feiras, sendo uma semana para planejamento das atividades e a outra para execução das ações no grupo. Foram utilizadas diversas metodologias de trabalho: rodas de conversas, dinâmicas de grupo e exposições dialogadas.

Os encontros foram realizados da seguinte forma:

- $1^{\circ}$ encontro: Levantamento de demandas dos participantes

Nesse primeiro momento, foi realizada uma dinâmica de apresentação, descritos os objetivos dos encontros e elaborado coletivamente o calendário das atividades com temáticas de interesse dos apenados.

- $2^{\circ}$ encontro: Acesso aos serviços de saúde

O objetivo desse encontro foi mostrar os serviços de saúde disponíveis no município, a função de cada um e a forma de acesso. Através de tarjetas de diferentes cores, distribuídas entre os participantes, foi construído um painel, contendo o nome do ponto de atenção à saúde, as demandas atendidas nesses locais e as formas de acesso.

- $3^{\circ}$ encontro: Cuidar da saúde mental

Nesse encontro, o psicólogo da equipe de residentes falou sobre ansiedade, depressão e uso de drogas. Foi realizada a escuta dos participantes e o compartilhamento de atividades ocupacionais, para evitar a ociosidade da mente e, assim, proporcionar uma melhor fluidez psicológica. No momento, os participantes pediram a realização de algum teste de mensuração, sendo assim aplicado o Teste de Identificação de Distúrbio de Uso de Álcool (Audit).

O Audit é composto por dez perguntas que investigam o padrão de uso de álcool nos últimos 12 meses. Cada resposta gera uma pontuação. Da $1^{\mathrm{a}}$ até a $8^{\mathrm{a}}$ questão, a pontuação varia de 0 a 4 pontos; as questões $9^{\mathrm{a}} \mathrm{e}$ $10^{\mathrm{a}}$ apresentam os valores 0,2 ou 4 pontos, a depender da resposta. $\mathrm{O}$ valor da soma das dez pontuações indica a presença e a intensidade dos problemas relacionados ao álcool16. Segue o quadro 1, com o questionário Audit. 
Quadro 1. Questionário do Teste de Identificação de Distúrbio de Uso de Álcool. Fortaleza, Ceará, 2019

1. Com que frequência consome bebidas que contêm álcool? [Escreva o número que melhor corresponde à sua situação].

$0=$ nunca

1 = uma vez por mês ou menos

2 = duas a quatro vezes por mês

$3=$ duas a três vezes por semana

4 = quatro ou mais vezes por semana

2. Quando bebe, quantas bebidas contendo álcool consome num dia normal?

$0=$ uma ou duas

$1=$ três ou quatro

$2=$ cinco ou seis

$3=$ de sete a nove

4 = dez ou mais

3. Com que frequência consome seis bebidas ou mais numa única ocasião?

$0=$ nunca

$1=$ menos de um vez por mês

2 = pelo menos uma vez por mês

$3=$ pelo menos uma vez por semana

4 = diariamente ou quase diariamente

4. Nos últimos 12 meses, com que frequência se apercebeu de que não conseguia parar de beber depois de começar?

$0=$ nunca

$1=$ menos de um vez por mês

2 = pelo menos uma vez por mês

3 = pelo menos uma vez por semana

4 = diariamente ou quase diariamente

5. Nos últimos 12 meses, com que frequência não conseguiu cumprir as tarefas que habitualmente lhe exigem por ter bebido?

$0=$ nunca

$1=$ menos de um vez por mês

2 = pelo menos uma vez por mês

3 = pelo menos uma vez por semana

4 = diariamente ou quase diariamente

6. Nos últimos 12 meses, com que frequência precisou beber logo de manhã para 'curar' uma ressaca?

$0=$ nunca

$1=$ menos de um vez por mês

2 = pelo menos uma vez por mês

3 = pelo menos uma vez por semana

4 = diariamente ou quase diariamente

7. Nos últimos 12 meses, com que frequência teve sentimentos de culpa ou de remorsos por ter bebido?

$0=$ nunca

$1=$ menos de um vez por mês

2 = pelo menos uma vez por mês

3 = pelo menos uma vez por semana

4 = diariamente ou quase diariamente

8. Nos últimos 12 meses, com que frequência não se lembrou do que aconteceu na noite anterior por ter bebido?

$0=$ nunca

$1=$ menos de um vez por mês

2 = pelo menos uma vez por mês

3 = pelo menos uma vez por semana

4 = diariamente ou quase diariamente 
Quadro 1. (cont.)

\section{Alguma vez já ficou ferido ou alguém ficou por você ter bebido? \\ $0=$ não \\ 1 = sim, mas não nos últimos 12 meses \\ 2 = sim, aconteceu nos últimos 12 meses}

10. Alguma vez um familiar, amigo, médico ou profissional de saúde já manifestou preocupação pelo seu consumo de álcool ou sugeriu que deixasse de beber?

$0=$ não

$1=$ sim, mas não nos últimos 12 meses

2 = sim, aconteceu nos últimos 12 meses

Fonte: Moretti-Pires e Corradi-Webster17.

Nota: Audit = teste para identificação de problemas relacionados ao uso de álcool: roteiro para uso em atenção primária.

- $4^{\circ}$ encontro: Saúde bucal

Foram explicados os principais agravos que atingem a cavidade bucal (cárie, gengivite, herpes e periodontite), através de imagens, e as formas de prevenção (uso de fio dental, escovação adequada). Na ocasião, foram distribuídas escovas de dente e realizada aplicação tópica de flúor.

- $5^{\circ}$ encontro: Alimentação saudável

Orientou-se sobre a importância do autocuidado para prevenir ou controlar as Doenças Crônicas Não Transmissíveis (DCNT), através de uma alimentação saudável e da prática de atividades físicas. Na ocasião, foi realizado o cálculo do Índice de Massa Corporal (IMC). O IMC é estimado pela relação entre o peso (em quilogramas) e a altura (em metros) do indivíduo, expresso em $\mathrm{kg} / \mathrm{m}^{2}$. O IMC classifica o indivíduo com relação ao peso e também é um indicador de riscos para a saúde ${ }^{\mathbf{1 8}}$.

- $6^{\circ}$ encontro: Prevenção de Infecções Sexualmente Transmissíveis (ISTs) e Síndrome da Imunodeficiência Humana Adquirida (Sida) e o uso do preservativo

Foi utilizado para embasamento teórico o 'Caderno de Atenção Básica n⿳0 18' (2006) ${ }^{19}$, do Ministério da Saúde, e explicado sobre prevenção, sinais e sintomas das ISTs, diagnóstico, tratamento, cura, reabilitação, e sobre a importância do uso do preservativo nas relações sexuais. Houve distribuição de panfletos explicativos, esclarecimento de dúvidas, quebra de tabus e entrega de preservativos masculinos.

- $7^{\circ}$ encontro: Encerramento das atividades

No último encontro, aconteceu uma confraternização natalina, com a presença das pessoas envolvidas nesse projeto: juíza e promotora municipal, professoras, residentes, voluntários que contribuíram para as atividades formativas, os apenados e seus familiares. Foi um momento bem gratificante e de emoções.

O presente estudo seguiu as recomendações da Resolução no ${ }^{\circ} 466 / 12$, do Conselho Nacional de Saúde. Essa Resolução incorpora os referenciais da bioética, autonomia, não maleficência, beneficência, justiça e equidade.

Os riscos com o desenvolvimento da pesquisa foram mínimos, visto que não foi realizada identificação de nenhum participante. Entretanto, adotaram-se medidas para minorar esses possíveis riscos, tais como: segurança quanto à confidencialidade e à privacidade, proteção da pessoa, a não utilização das informações e o sigilo.

\section{Resultados e discussão}

Foi realizado um total de sete encontros com os apenados, sendo o último encontro destinado ao encerramento do grupo. A frequência no grupo era variável entre os participantes. Existiam aqueles que não faltavam a nenhum encontro, mas também havia alguns com 
assiduidade menor, no entanto, as atividades em nenhum encontro deixaram de acontecer por ausência de participantes.

Iniciou-se com uma dinâmica de apresentação, na qual cada um dizia seu nome e uma qualidade com a primeira letra do nome. Esse foi um momento descontraído para romper com a ansiedade, que serviu para 'quebrar o gelo' e diminuir a distância entre os profissionais e os apenados, ajudando na integração e na manutenção do senso de equipe, tão essencial para uma construção coletiva com resultados positivos. Falou-se também do objetivo de estar ali para a realização de ações de promoção e educação em saúde, e eles foram consultados se consideravam importante a contribuição de cada categoria profissional. Com a aceitação do grupo, foram perguntados se gostariam de adquirir conhecimentos, de modo que foi elaborado um calendário com a temática dos encontros.

No segundo encontro, os participantes construíram juntos um painel com os principais pontos da rede de atenção à saúde: UBS, hospital, UPA, policlínica, CEO, Caps II, Caps AD e Samu. Observou-se o desconhecimento de alguns sobre a existência e as funções dos serviços no município, além do fato de alguns deles também não possuírem o Cartão Nacional de Saúde (CNS). O CNS é o documento que possibilita vincular os procedimentos, ações e serviços de saúde executados no SUS ao cidadão, ao profissional de saúde que os realizou e também à unidade de saúde onde foram realizados. Foi explicado o que é necessário para solicitar o documento. Na ocasião, é solicitada a apresentação de documento (não obrigatória) que permita a identificação do usuário do SUS. Essas informações são colhidas na Secretaria Municipal de Saúde, em postos de saúde, ou através do agente de saúde. Na oportunidade, foi ofertado o CNS para aqueles que desejassem possuir.

Ainda ressalta-se a deficiência com relação às orientações tanto relacionadas ao funcionamento dos serviços de saúde existentes ou sobre os tipos de atendimento que podem ser prestados pela equipe da UBS e/ou em quais situações a equipe encaminha para atendimento em outras unidades da rede pública de saúde; quanto com relação às orientações acerca dos cuidados em saúde. No tocante à assistência à saúde, vários estudos ainda mostram a insuficiência nos cuidados ofertados, quando não se tem consultas de admissão nem tampouco periódicas, apenas de maneira pontual e que atendam casos de urgência e quando não são realizados exames laboratoriais ${ }^{20}$.

Torna-se necessário ajustar os espaços físicos das unidades de saúde para que os profissionais executem suas funções de maneira mais adequada, com atenção especial para o acesso da população penitenciária. Questões organizacionais, como o cadastramento da população carcerária nos estabelecimentos de saúde, devem ser melhoradas e atualizadas para que se possa atingir com eficiência o conhecimento, a prevenção, o controle e o tratamento de doenças no âmbito do encarceramento, bem como desenvolver ações de promoção da saúde e assistência direcionadas para os problemas mais frequentes da população carcerária ${ }^{7}$.

$\mathrm{Na}$ abordagem acerca das principais situações que representam o sofrimento mental comum, como as síndromes depressivas, ansiosas e de somatização (queixas físicas sem explicação médica), percebeu-se que alguns participantes estavam se identificando com sinais e sintomas (ex: tristeza, desânimo, insônia, falta de perspectiva, ansiedade, solidão etc.) das síndromes destacadas pelo psicólogo. Houve relatos de como é sentir-se cumprindo ordem judicial, sobre as dificuldades enfrentadas para reconquistar a confiança dos familiares, reconstruir os vínculos e também para conseguir emprego.

A literatura corrobora as manifestações clínicas evidenciadas pelos apenados quando traz que a ocorrência de algum acontecimento marcante na vida aumenta o risco de sofrimento mental. Pesquisas mostraram que, na maioria das vezes, o que torna esses acontecimentos marcantes é o desencadeamento de sentimentos de humilhação ou de sentir-se sem saída ${ }^{16}$. 
A humilhação está normalmente associada à perda de um vínculo importante, uma separação conjugal, um ato de delinquência ou, ainda, a situações que são vividas, como uma diminuição da pessoa diante da sua comunidade. Já a sensação de sentir-se sem saída foi relacionada a eventos que de alguma forma confirmam a impossibilidade de mudar uma situação vivida como punitiva ${ }^{16}$. No estudo de Audi et al. 21, a prevalência de Transtorno Mental Comum (TMC) foi de 66,7\%.

Na aplicação do Audit, foi identificado o grau de dependência de cada um quanto ao uso de álcool, e a partir daí foram dados os devidos encaminhamentos de acordo com os escores obtidos. Aqueles que obtiveram a pontuação entre zero e 15, o que representou a maioria deles, foram orientados quanto aos serviços ofertados pelas equipes das UBS. Do total dos que responderam ao questionário, três apresentaram pontuação entre 16 e 40, mostrando necessidade de uma intervenção realizada pelo serviço especializado, sendo, portanto, encaminhados para o Caps AD. Cada participante ficava com o seu próprio questionário, com seus resultados obtidos.

As PPL no País têm maior vulnerabilidade social, devido à posição que ocupam na sociedade. Suas dificuldades assumem proporção maior em vários aspectos, com acesso restrito a bens e serviços, como os relacionados à educação, à atenção à saúde e poucas oportunidades de trabalho, por exemplo20.

Durante a realização de cada encontro, os participantes apresentaram muitos questionamentos, dúvidas e receios frente às condições do regime semiaberto. Eles aparentavam ter percepções limitadas sobre a assistência à saúde, principalmente por esta ser pontual, quando em casos de doença ou de sua permanência. Os maiores questionamentos eram acerca de ações de saúde básicas, tais como necessidade de continuidade e atualização de vacinas, e higiene íntima e oral, bons hábitos alimentares e os riscos que o etilismo, o tabagismo e o abuso de outras drogas podem ocasionar em curto e longo prazo.
No quarto encontro, foi realizada a atividade sobre a saúde bucal. A maioria dos participantes relatou que há muito tempo não realizaram uma consulta odontológica. A partir da explicação da dentista, todos reconheceram a importância da procura do profissional mesmo diante da inexistência de algum sinal de anormalidade, pois a melhor maneira para evitar complicações futuras é através de ações preventivas. No momento, foi ofertada para todos os presentes a distribuição de escovas de dentes e realizada a aplicação tópica de flúor, uma vez que muitos deles não recordavam a última vez que tinham realizado a aplicação e desconheciam a importância disso.

Existe carência de profissionais médicos, psicólogos, dentistas e técnicos de enfermagem, e a maneira muito precária com que os cuidados preventivos e curativos, previstos em lei, são oferecidos aos presos agrava as condições de saúde, potencializando os sintomas físicos e mentais. A prestação de serviços de saúde nos cárceres do Estado é de flagrante descumprimento do que é prescrito na PNAISP8.

Segundo relatos informais de algumas das populações privadas de liberdade, a assistência à saúde na unidade prisional restringe-se basicamente a campanhas, na solicitação de alguns exames, tais como: anti-HIV, para tuberculose, exames de rotina e para infecções sexualmente transmissíveis; na realização de algumas vacinas e na administração de medicamentos em caso de doenças sintomáticas ou quando passam mal.

O quinto encontro foi destinado para as questões voltadas para a importância das práticas do autocuidado (alimentação saudável e prática de atividade física). Foi realizado o cálculo do IMC dos que estavam presentes. Para o cálculo do IMC, foi necessária a utilização de balança digital para verificação de peso e de fita métrica para obtenção da altura. A maioria dos participantes estava com peso adequado para sua altura, com o IMC entre 18,5 e 24,9), mesmo referindo a não restrição no consumo de alimentos ricos em gorduras, calóricos e pouco nutritivos do ponto de 
vista nutricional. Não foi identificado nenhum participante com obesidade ou com relato de tratamento para problemas de hipertensão e/ ou diabetes. Dos participantes, apenas três referiram a realização regular de atividade física.

Em estudo realizado com mulheres encarceradas, foi encontrado que $70 \%$ não praticavam atividade física, 45,5\% classificadas como eutróficas e a maioria delas apresentava sobrepeso ou obesidade (56,9\%). Com relação à presença de doenças crônicas não transmissiveis, foi encontrada uma prevalência de $21,4 \%$ de hipertensão arterial e 3,2\% de diabetes mellitus ${ }^{21}$.

No penúltimo encontro, falou-se sobre a importância do uso do preservativo e da prevenção de Infecções Sexualmente Transmissíveis (ISTs) e do HIV. Notou-se um certo receio e, ao mesmo tempo, curiosidade dos homens quanto à abordagem dessa temática. Eles faziam perguntas acerca da forma de transmissão, dos sintomas, de como se dava o tratamento e se tinha cura. Foram explicados aspectos gerais sobre as principais ISTs, tais como: herpes genital, sífilis, gonorreia, infecção pelo papilomavírus humano/HPV, hepatites virais (B e C); e também foram mencionados os testes rápidos que são realizados nas UBS. Na ocasião, não foi possível a realização dos testes rápidos, pois, para todas as intervenções realizadas, era necessário o envio prévio da descrição da ação para avaliação e autorização judicial, portanto, a equipe realizou somente a educação em saúde.

Assim, os relatos verbais indicam que a maior preocupação no grupo de detentos, além do tempo de detenção, é o adoecimento e o acesso ao tratamento e à cura, já que as condições de confinamento vivenciadas pela população deste relato de experiência demonstram dificuldades para o controle das doenças identificadas, bem como para a realização de medidas para o tratamento eficaz.

Ressalta-se, ainda, que o medo de descobrir doenças incuráveis ou infectocontagiosas é um forte impedimento observado nessas pessoas, além de uma má adesão às orientações dos profissionais da saúde quando estes realizam consultas. Infere-se, ainda, pela vivência e escuta durante os encontros que a condição de encarceramento, o medo de rejeição e o estigma pela condição de detento aumentam as vulnerabilidades e diminuem as chances dessas pessoas de se identificarem como objeto de cuidado em saúde.

A superlotação e a falta de avaliação para adoção de medidas ininterruptas de assistência, prevenção e promoção de saúde para as PPL podem piorar os quadros anteriores e desenvolver doenças, bem como pode ocorrer a transmissão de doenças infecciosas intrainstitucionais ${ }^{20}$. Dada a vulnerabilidade às doenças infectocontagiosas, identificamos a necessidade de a população encarcerada ter acesso às ações de atenção à saúde em todos os níveis de complexidade ${ }^{22}$.

No desenvolvimento de todas as ações, foi valorizado o conhecimento que já possuíam, e, dessa forma, foi possível viabilizar uma melhor sistematização do conhecimento adquirido nas variadas abordagens que compõem o cuidado à saúde da pessoa encarcerada, deixando claro ao grupo que apenas lhes faltava estabelecer o elo entre o saber e o fazer da promoção da saúde, possibilitando, assim, o desenvolvimento de uma consciência crítico-reflexiva com a finalidade de transformação do sujeito inserido no contexto social"1.

Ademais, foi possível perceber nos encontros que os detentos em regime semiaberto percebem a assistência à saúde de forma positiva, quando estas são bem conduzidas e aqueles são valorizados enquanto sujeitos que necessitam estar contemplados nos cuidados à saúde.

O término do período letivo coincidiu com o fim das atividades no grupo, e o sentimento gerado foi de satisfação, tanto pela possibilidade de promover ações educativas para essas pessoas como pelo convite recebido para participar do encerramento do ano com a realização de uma confraternização natalina. A turma dos residentes foi bastante elogiada pela iniciativa e recebeu mensagens de agradecimento da juíza municipal, das professoras do projeto e 
também dos apenados, o que deixou claro o quanto foi importante a educação em saúde no empoderamento dos indivíduos para o seu autocuidado, garantia da autonomia e promoção da sua saúde.

De acordo com Salci ${ }^{23}$, os profissionais de enfermagem são fundamentais na construção de um contexto emancipatório, no respeito e na confiança das potencialidades dos seres humanos, com os quais interagem e pactuam parcerias nas ações de educação em saúde. Devem ter uma compressão ampliada das questões que envolvem a complexidade da temática educação em saúde e, dessa maneira, realizar ações emancipatórias de promoção da saúde que ultrapassem o modelo biomédico e atuem de forma participativa para que a pessoa obtenha conhecimento necessário para tomar decisões conscientes no seu processo saúde-doença e de viver saudável.

\section{Considerações finais}

Apesar da implantação de políticas públicas voltadas para a população privada de liberdade, ainda existe muita dificuldade em sua implementação. Observa-se um contexto ainda muito marcado por uma assistência em saúde precária ou até inexistente. É importante instigar discussões sobre saúde no sistema penitenciário nos centros educacionais formadores de recursos humanos para o SUS, tendo em vista a apreensão de conhecimentos e o desenvolvimento de habilidades e competências, a fim de contribuir para a adequada atuação dos profissionais de enfermagem e demais trabalhadores da saúde nesse cenário.

É fundamental que mais estudos sejam feitos para mostrar a realidade dessa população, visto que essa temática constitui-se, ainda, um campo pouco explorado, permitindo, também, a conscientização dos profissionais de enfermagem e das demais categorias profissionais acerca da necessidade e da importância de uma saúde mais igualitária e equânime, independentemente da população à qual ela seja prestada.

Destarte, pode-se considerar que as práticas produzidas pela equipe de residentes junto aos homens que cumprem regime semiaberto permitiram o desenvolvimento de ações educativas e de promoção da saúde que proporcionaram maior empoderamento e autonomia dessas pessoas nas questões relativas à sua saúde.

\section{Colaboradores}

Serra AEG (0000-0002-0705-3055)* contribuiu para a concepção, o planejamento e para a revisão crítica do conteúdo. Lima RCRO (0000-0002-7989-5104)* contribuiu para o planejamento, a análise e a interpretação dos dados; revisão crítica do conteúdo; e aprovação da versão final. 


\section{Referências}

1. Corte Interamericana de Derechos Humanos. Cuadernillo de jurisprudencia de la Corte Interamericana de Derechos Humanos $n^{\circ}$ 9: personas privadas de libertad. San José: Corte Interamericana de Derechos Humanos; 2017.

2. Damas FB. Assistência e condições de saúde nas prisões de Santa Catarina, Brasil. Rev. saúde pública. 2012; 5(3):6-22.

3. Brasil. Lei $\mathrm{n}^{0} 7210$, de 11 de julho de 1984. Institui a Lei de Execução Penal. Diário Oficial da União. 13 Jul 1984.

4. Gois SM, Junior HPOS, Silveira MFA, et al. Para além das grades e punições: uma revisão sistemática sobre a saúde penitenciária. Ciênc. Saúde Colet. [internet]. 2012 [acesso em 2019 jan 19]; 17(5):1235-1246. Disponível em: http://www.scielo.br/pdf/csc/v17n5/ a17v17n5.pdf.

5. Brasil. Ministério da Saúde. Portaria $n^{0} 1.777$, de 09 de setembro de 2003. Aprovar o Plano Nacional de Saúde no Sistema Penitenciário, constante do ANEXO I desta Portaria, destinado a prover a atenção integral à saúde da população prisional confinada em unidades masculinas e femininas, bem como nas psiquiátricas. Diário Oficial da União. 9 Set 2009.

6. Brasil. Ministério da Saúde. Portaria Interministerial $\mathrm{n}^{\mathrm{o}}$ 1, de 2 de janeiro de 2014. Institui a Política Nacional de Atenção Integral à Saúde das Pessoas Privadas de Liberdade no Sistema Prisional (PNAISP) no âmbito do Sistema Único de Saúde (SUS). Diário Oficial da União. 2 Jan 2014.

7. Barbosa ML, Celino SDM, Oliveira, LV, et al. Atenção básica à saúde de apenados no sistema penitenciário: subsídios para a atuação da enfermagem. Esc. Anna Nery Rev. Enferm. [internet]. 2014 [acesso em 2019 jan 19]; 18(4):586-592. Disponível em: http://www. scielo.br/pdf/ean/v18n4/1414-8145-ean-18-04-0586. pdf.

8. Minayo MC, Ribeiro AP. Condições de saúde dos pre- sos do estado do Rio de Janeiro, Brasil. Ciênc. Saúde Colet. [internet]. 2016 [acesso em 2019 jan 18]; 21(7):2031-2040. Disponível em: http://www.scielo. br/scielo.php?pid=S1413.

9. Santa Catarina. Governo do Estado, Secretaria de Estado da Saúde, Secretaria de Estado da Justiça e Cidadania. Manual: Atribuições da Equipe de Saúde no Sistema Prisional. Santa Catarina: Secretaria de Estado da Justiça e Cidadania; 2016. 32 p.

10. Brasil. Ministério da Saúde. Portaria n ${ }^{\circ} 2.436$, de 21 de setembro de 2017. Aprova a Política Nacional de Atenção Básica, estabelecendo a revisão de diretrizes para a organização da Atenção Básica, no âmbito do Sistema Único de Saúde (SUS). Diário Oficial da União. 22 Set 2017.

11. Menezes PR, Lopes L G, Vasconcelos CTM, et al. Promoção da saúde em unidade prisional: relato de experiência. In: Anais do $4^{\circ}$ Encontro Internacional de Jovens Investigadores; 2017 Set 4; Fortaleza. Fortaleza: UNEB; 2017. p. 1-11.

12. Minayo MCS. O desafio do conhecimento. Pesquisa qualitativa em saúde. 12. ed. São Paulo: Hucitec; 2010.

13. Marconi MA, Lakatos EM. Metodologia do Trabalho Científico. 8. ed. São Paulo: Atlas; 2017.

14. Allison T, Peter S, Jonathan C. Critérios consolidados para relato de pesquisa qualitativa (COREQ): lista de verificação de 32 itens para entrevista e grupos focais. Rev. Inter. Qual. Assist. Saúde. 2017;19:349-357.

15. Heimann C, Prado C, Moraes RRSP, et al. A construção do conhecimento da enfermagem baseada no método construtivista. Rev. Esc. Enferm. USP. 2013; 47(4):997-1000.

16. Brasil. Ministério da Saúde, Secretaria de Atenção à Saúde, Departamento de Atenção Básica. Saúde Mental. Cadernos de Atenção Básica - n 34. Brasília, DF: Ministério da Saúde; 2013. 
17. Moretti-Pires RO, Corradi-Webster CM. Adaptação e validação do Alcohol Use Disorder Identification Test (AUDIT) para população ribeirinha do interior da Amazônia, Brasil. Cad. Saúde Pública [internet]. 2011 [acesso em 2020 jan 31]; 27(3):497-509. Disponível em: http://dx.doi.org/10.1590/S0102-311X2011000300010.

18. Brasil. Ministério da Saúde, Secretaria de Atenção à Saúde, Departamento de Atenção Básica. Estratégias para o cuidado da pessoa com doença crônica: obesidade. Cadernos de Atenção Básica - no 12. Brasília, DF: Ministério da Saúde; 2014.

19. Brasil. Ministério da Saúde, Secretaria de Atenção à Saúde, Departamento de Atenção Básica. HIV/Aids, hepatites e outras DST. Cadernos de Atenção Básica - $\mathrm{n}^{\circ}$ 18. Brasília, DF: Ministério da Saúde; 2006.

20. Valim EMA, Daibem AML, Hossne WS. Atenção à saúde de pessoas privadas de liberdade. Rev. bioética. 2018; 26(2):282-290.

21. Audi CAF, Santiago SM, Andrade MGG, et al. Inquérito sobre condições de saúde de mulheres encarceradas. Saúde debate. 2016; 40(109):112-124.

22. Mourão LF, Oliveira LB, Marques ADB, et al. Promoção da Saúde de mulheres encarceradas: um relato de experiência. SANARE. 2015; 14(1):52-57.

23. Salci MA, Maceno P, Rozza SG, et al. Educação em Sáude e suas perpectivas teóricas: algumas reflexões. Texto \& contexto enferm. 2013; 22(1):224-230.

Recebido em 08/08/2019 Aprovado em 29/10/2019

Conflito de interesses: inexistente

Suporte financeiro: não houve 